\title{
Interference Considerations for the Time Division Duplex Mode of the UMTS Terrestrial Radio Access
}

\author{
Harri Holma, Sanna Heikkinen, Otto-Aleksanteri Lehtinen, and Antti Toskala
}

\begin{abstract}
The air interface of the UMTS Terrestrial Radio Access (UTRA) covers both a frequency division duplex (FDD) part for the paired bands and a time division duplex (TDD) part for the unpaired bands of the UMTS spectrum. Universal Mobile Telecommunication System (UMTS) is the 3rd-generation mobile communication system. This paper presents an interference evaluation of the UTRA TDD. Since both uplink and downlink share the same frequency in TDD, the signals of the two transmission directions can interfere with each other. This interference can occur between two mobile stations or between two base stations within one carrier or between two operators. The interference between uplink and downlink is evaluated by system simulations in this paper. Synchronization and coordination requirements of UTRA TDD are evaluated based on the results.
\end{abstract}

Index Terms-Network synchronization, time division duplex (TDD), UMTS Terrestrial Access (UTRA).

\section{INTRODUCTION}

$\mathbf{T}$ HE SPECTRUM allocation for UMTS is shown in Fig. 1: for UTRA FDD $2 \times 60 \mathrm{MHz}$ and for UTRA TDD $15+$ $20 \mathrm{MHz}$ are allocated. Both FDD and TDD modes are needed to fully utilize the existing spectrum. In ETSI, the air interface for the 3rd-generation system (UMTS Terrestrial Access, UTRA) is WCDMA for the paired FDD (frequency division duplex) bands and TD/CDMA for the unpaired TDD (time division duplex) bands. UTRA FDD is suitable for large coverage areas, and it is also able to provide bit rates up to $2 \mathrm{Mb} / \mathrm{s}$ in small cells. A detailed description of the WCDMA FDD networks and their planning can be found in [1]. UTRA TDD is particularly well suited for small cells and for asymmetrical traffic. Asymmetrical traffic refers here to the case where the total capacity need for uplink and for downlink is different. The FDD and TDD modes have been harmonized to a large degree during the standardization work in ETSI and later in the 3rd-Generation Partnership Project (3GPP) to ease a dual mode implementation. 3GPP has combined the standardization efforts from several standardization organizations and the term UTRA in 3GPP stands for Universal Terrestrial Radio Access. The 3GPP specifications are available from [2]. The main features of the UTRA TDD mode are presented in this issue in [3].

In all TDD systems, uplink and downlink share the same frequency and, therefore, the signals of the two transmission directions can interfere with each other. The interference between

Manuscript received August 1999; revised February 28, 2000.

H. Holma and A. Toskala are with Nokia Networks, FIN-00045 Nokia Group, Finland (e-mail: harri.holma@nokia.com).

S. Heikkinen is with the University of Oulu, Telecommunications Laboratory, FIN-90571 Oulu, Finland.

O.-A. Lehtinen is with Nokia Mobile Phones, FIN-24101 Salo, Finland.

Publisher Item Identifier S 0733-8716(00)06107-2. uplink and downlink signals can occur between two mobile stations or between two base stations within one carrier or between two operators. This interference is evaluated by system simulations in the paper. Synchronization and coordination requirements of UTRA TDD are evaluated based on the results.

The paper is organized as follows. The main differences between FDD and TDD operation are introduced in Section II, in general, and between UTRA FDD and TDD physical layers in Section III. The possible interference situations between uplink and downlink in TDD are described in Section IV. The system simulation platform is introduced in Section V, followed by the analysis of the simulation results within one operator's band in Section VI, and between two operators in Section VII. Finally, the summary of the results is presented in Section VIII.

\section{TIME DIVISION DUPLEX OPERATION}

There are a few characteristics that are typical of TDD systems and different from the characteristics of FDD systems. Those characteristics are listed briefly below, and they apply also for UTRA TDD and FDD.

- Utilization of the unpaired band. The TDD system can be implemented on an unpaired band, while the FDD system always requires a pair of bands. In the future it is more likely that unpaired spectrum resources are cleared for UMTS, because no pair of bands, with reasonable duplex distance, is required for the TDD operation.

- Possible interference between uplink and downlink. Since both uplink and downlink share the same frequency in TDD, the signals of the two transmission directions can interfere with each other.

- Flexible capacity allocation between uplink and downlink. In TDD operation, uplink and downlink are divided in the time domain. It is possible to change the duplex switching point and move capacity from uplink to downlink, or vice versa, if the capacity requirement is asymmetric between uplink and downlink.

- Discontinuous transmission. The mobile and the base station transmission are discontinuous in TDD. The discontinuous transmission sets requirements on the implementation. Switching between transmission directions requires time, and the switching transients must be controlled. To avoid the overlapping of uplink and downlink transmissions, a guard period is used in the end of each slot.

- Reciprocal channel. The fast fading depends on the frequency and, therefore, in FDD systems the fast fading is uncorrelated between uplink and downlink. As the same 


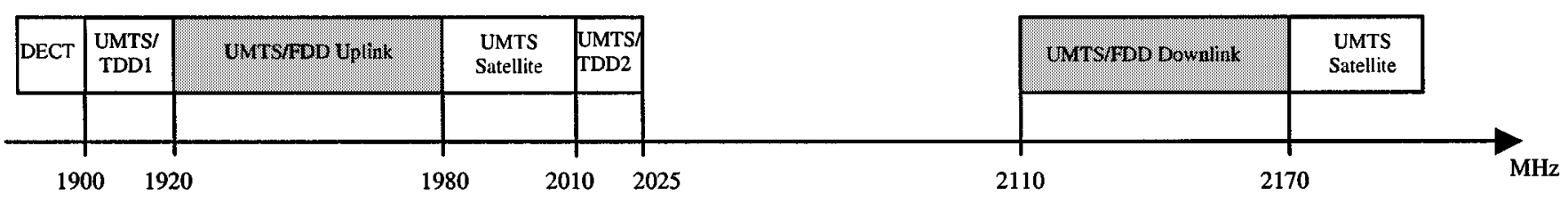

Fig. 1. UMTS spectrum allocation.

TABLE I

COMPARISON OF UTRA TDD AND FDD PHYSICAL LAYER KEY PARAMETERS

\begin{tabular}{l|c|c}
\hline & UTRA TDD & UTRA FDD \\
\hline $\begin{array}{l}\text { Multiple access } \\
\text { method }\end{array}$ & $\begin{array}{c}\text { TDMA, CDMA, (inherent } \\
\text { FDMA) }\end{array}$ & CDMA, (inherent FDMA) \\
\hline Multirate concept & $\begin{array}{c}\text { multicode, multislot and } \\
\text { orthogonal variable spreading } \\
\text { factor (OVSF) }\end{array}$ & multicode and OVFS \\
\hline Channel allocation & $\begin{array}{c}\text { slow and fast dynamic } \\
\text { channel allocation (DCA) } \\
\text { supported }\end{array}$ & no DCA required \\
\hline $\begin{array}{l}\text { Capacity allocation } \\
\text { between uplink and } \\
\text { downink }\end{array}$ & $\begin{array}{c}5 \mathrm{MHz} \text { carrier divided } \\
\text { between uplink and downlink. } \\
\text { Downlink / uplink capacity } \\
\text { can be adjusted between } \\
2-14 \text { out of 15 slots. }\end{array}$ & $5 \mathrm{MHz}$ for downlink \\
\hline
\end{tabular}

frequency is used both for uplink and downlink in TDD, the fast fading is the same in uplink and in downlink. Based on the received signal, the TDD transceiver can estimate the fast fading which will affect its transmission. The knowledge of the fast fading can be utilized in power control and in adaptive antenna techniques in TDD.

\section{UTRA TDD PHYSICAL LAYER}

The physical layer of UTRA TDD is a combination of TDMA and CDMA access methods (see [3]). The $10 \mathrm{~ms}$ frame is divided into 15 time slots, each of which can support parallel CDMA spreading codes with a maximum spreading factor of 16. The channel estimation is based on the midamble code. Table I presents a summary of the UTRA physical layer parameters that are relevant for the interference evaluation.

\section{INTERFERENCE BETWEEN UPLINK AND DOWNLINK IN TDD}

\section{A. TDD Interference}

Since both uplink and downlink share the same frequency band in TDD, the uplink and downlink signals can interfere with each other. This kind of interference occurs if the base stations are not synchronized. The interference can be present also if the division between uplink and downlink is different in adjacent cells, even if the base stations are frame synchronized. The interference between uplink and downlink occurs between two mobile stations and between two base stations. In FDD operation, the duplex separation prevents the interference between uplink and downlink. The interference between uplink and downlink in TDD is studied also in [4] and [5].

The uplink and downlink signals on adjacent frequencies can interfere with each other and, therefore, the interference between uplink and downlink signals can take place also between two operators.
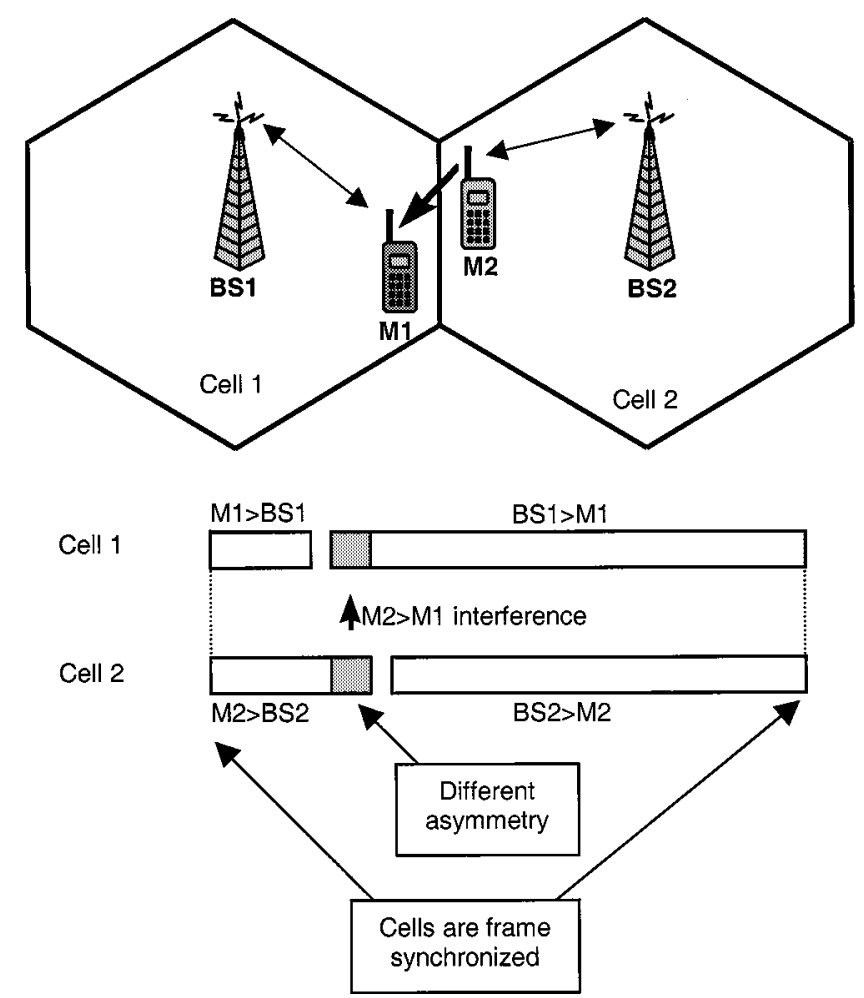

Fig. 2. Mobile-to-mobile interference at the cell border.

\section{B. Mobile-to-Mobile Interference}

A case with mobile-to-mobile interference is shown in Fig. 2 where the mobile M2 is transmitting and the mobile M1 is receiving in the same or adjacent frequency bands in adjacent cells. The cells here are frame synchronized, but a different asymmetry between uplink and downlink is used in those two cells. Mobile-to-mobile interference is statistical because the locations of the mobiles cannot be controlled. Therefore, mobile-to-mobile interference cannot be avoided by network planning.

\section{Base Station-to-Base Station Interference}

Base station-to-base station interference occurs if a base station is transmitting and another base station is receiving in the same or adjacent frequency bands in adjacent cells. Base station-to-base station interference depends heavily on the path loss between the two base stations and, therefore, this interference case can be controlled by network planning. This interference can occur within one operator's band and between different operators' base stations. The interference scenario within one operator's band in shown in Fig. 3 where the base stations in adjacent cells are interfering each other. In the case of different 


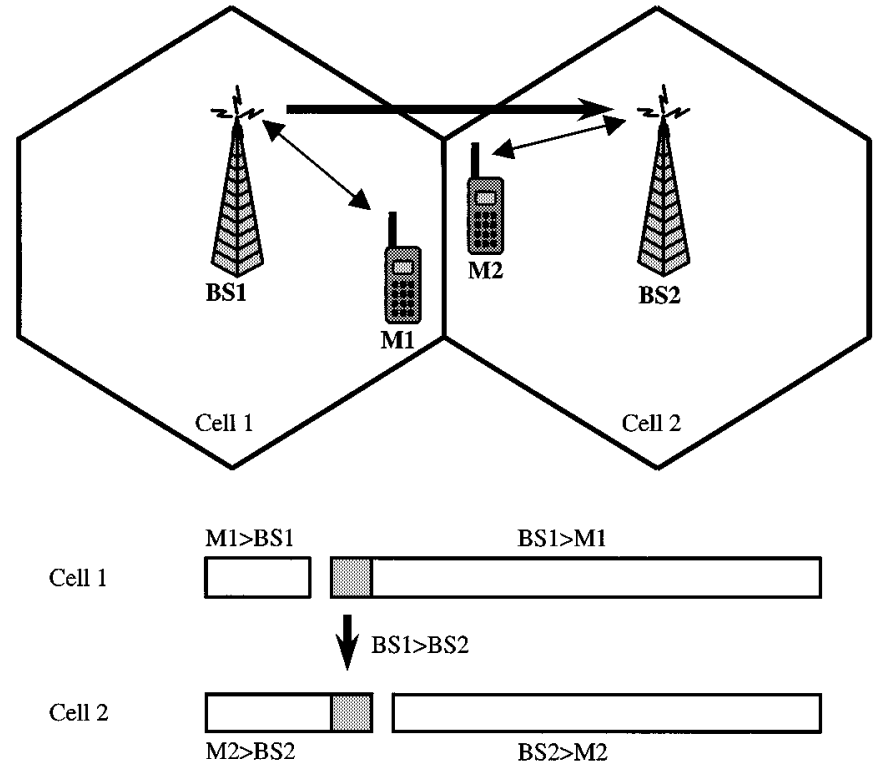

Fig. 3. Base station-to-base station interference between adjacent cells.

TABLE II

THE EFFECTS OF $t_{\text {off }}$

\begin{tabular}{l|c|c|c|c}
\hline & $\begin{array}{c}\text { BS-to-BS } \\
\text { interference }\end{array}$ & $\begin{array}{c}\text { MS-to-MS } \\
\text { interference }\end{array}$ & $\begin{array}{c}\text { BS-to-MS } \\
\text { Interference }\end{array}$ & $\begin{array}{c}\text { MS-to-BS } \\
\text { interference }\end{array}$ \\
\hline small $t_{\text {off }}$ & low & low & high & high \\
\hline large $t_{\text {ott }}$ & high & high & low & low \\
\hline
\end{tabular}

operators, the base stations can be closer to each other or even collocated.

\section{SimUlation PlatForm}

A simulation platform was generated to evaluate the interference scenarios in TDD operation. Operation during one time slot is considered. It is assumed that the time slots in different cells have some time offset $t_{\text {off }}$. The effects of $t_{\text {off }}$ are summarized in Table II. Small $t_{\text {off }}$ corresponds to the case where the uplink transmissions occur at the same time in adjacent cells, and no overlapping of uplink and downlink occurs. Small $t_{\text {off }}$ leads to low base station-to-base station (BS-to-BS) and mobile-to-mobile (MS-to-MS) interference, but higher mobile-to-base station (MS-to-BS) and base station-to-mobile (BS-to-MS) interference. Conversely, increasing $t_{\text {off }}$ results in higher BS-to-BS and MS-to-MS interference and lower MS-to-BS and BS-to-MS interference.

With $t_{\mathrm{off}}$ as the absolute frame offset and $t_{\text {slot }}$ the duration of one time slot, the frame synchronization error is given by the ratio

$$
\alpha=t_{\mathrm{off}} / t_{\mathrm{slot}}
$$

The effects of the frame synchronization error $\alpha$ were evaluated as shown in Fig. 4. Network $b$ is the source and network $a$ is the sink of the interference. The value of $\alpha$ defines the relation between the interference caused by mobiles and the interference caused by base stations during the time slot under consideration.

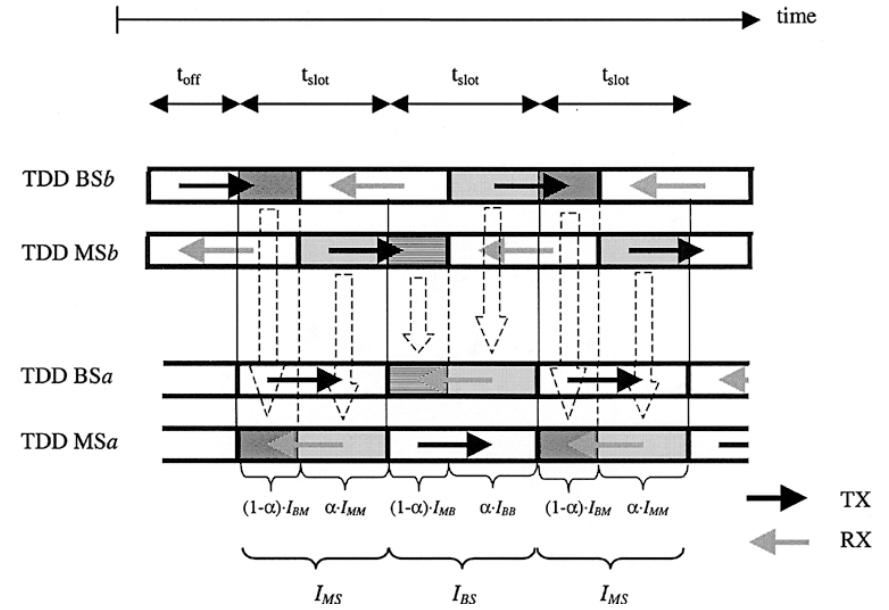

Fig. 4. TDD Interference. $I_{\mathrm{BS}}=$ Interference level at base station, $I_{\mathrm{MS}}=$ Interference level at mobile, $I_{\mathrm{MB}}=$ Mobile-to-base station interference, $I_{\mathrm{BM}}=$ Base station-to-mobile interference. $I_{\mathrm{MM}}=$ Mobile-to-mobile interference, $I_{\mathrm{BB}}=$ Base station-to-base station interference.

In order to model nonsynchronized frames or different asymmetries, the method used in [6] was adopted.

All interference caused by the other users in the cell of interest and the other cells is considered as an increase in the noise level at the receiver. Ideal power control is assumed, thus all desired signals are received at the same power level at the base station. The bit energy-to-noise density ratio is therefore given by

$$
\frac{E_{b}}{N_{0}}=\frac{P_{r x} \cdot \mathrm{PG}}{P_{r x}(M-1)+\eta}
$$

where

$P_{r x} \quad$ signal power received from the desired user;

$M \quad$ number of users;

$\eta \quad$ thermal noise power;

PG processing gain.

Equation (2) assumes a conventional matched filter receiver. A joint detection receiver would cancel part of the intra-cell interference between mobile and base station, but it would not cancel inter-cell interference between two mobile stations or between two base stations. In theory, a joint detection receiver could also try to remove inter-cell interference, but that is not considered feasible from the implementation point of view with today's technology, neither does the UTRA TDD network provide signalling information of necessary parameters used in other cells or by other mobile stations. Therefore, for simplicity, a joint detection receiver is not considered here.

Solving (2) for $M$ yields

$$
M_{0}=\frac{\mathrm{PG}}{\frac{E_{b}}{N_{0}}}-\frac{\eta}{P_{r x}}+1
$$

where $M_{0}$ denotes the single cell capacity without the contribution of the additional inter-cell interference. The inter-cell interference can originate from inter-cell mobiles or from neighboring base stations. 


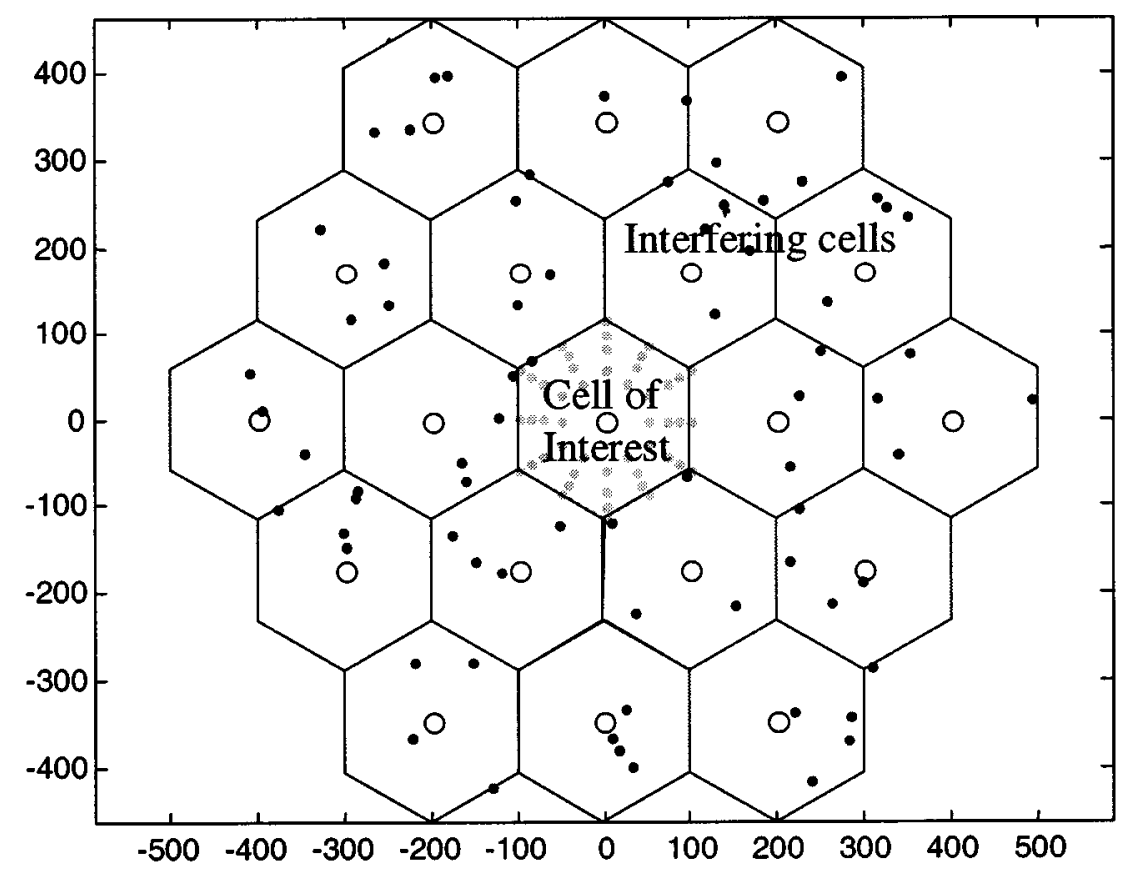

Base station

- Mobile station

Fig. 5. Simulation scenario for TDD $\rightarrow$ TDD cochannel interference.

The uplink capacity is expressed in terms of the remaining capacity given by the ratio [6]

$$
\frac{M}{M_{0}}=1-\frac{1}{P_{r x}\left(1+\frac{\mathrm{PG}}{\frac{E_{b}}{N_{0}}}\right)-\eta} .
$$

$M / M_{0}=1$ indicates that the additional inter-cell interference does not reduce the capacity in the cell of interest, whereas $M / M_{0}=0$ means that the capacity is completely destroyed in the time slot under consideration.

For downlink capacity calculations, a mobile is placed in one of the points of interest where the interference from the adjacent cells was calculated. The transmission power of the base station is set to combat the highest attenuation within the middle cell. The received signal level at the mobile is then obtained from the path loss. It is assumed that the maximum number of users in one time slot is 8, which is fed to (2) and the $E_{b} / N_{0}$ value for that mobile is found. The value obtained is then compared to the required $E_{b} / N_{0}$, and if this $E_{b} / N_{0}$ level is not achieved, an outage at that point is caused. The process is then repeated over all points of interest, which are shown in the middle cell in Fig. 5.

The scenario used for the cochannel interference investigation is shown in Fig. 5. The cell of interest with an inner radius $100 \mathrm{~m}$ is in the middle. It is surrounded by two tiers (six cells in the inner and twelve in the outer tier) of interfering cells of inner radius $100 \mathrm{~m}$. The cells are arranged so that they produce continuous coverage but do not overlap. It is assumed that cells beyond the second tier around the center cell do not contribute significantly to the interference levels.

The assumed propagation model is the COST 231 indoor office model without floor losses, and is given by

$$
L(\mathrm{~dB})=37+30 \log (d)
$$

where $d$ is the transmitter-receiver distance in meters. Lognormal shadowing with $\sigma=10 \mathrm{~dB}$ is assumed.

\section{INTRA-OPERATOR INTERFERENCE EVALUATION}

The simulation results for a cochannel interference scenario with a bit rate of $16 \mathrm{~kb} / \mathrm{s}$ are presented below. The bit rate of $16 \mathrm{~kb} / \mathrm{s}$ assumes a spreading factor of 8 and, therefore, the maximum number of $16-\mathrm{kb} / \mathrm{s}$ users per slot is 8 . The values of the synchronization error $\alpha$ as well as the number of users in the interfering time slots are varied. $\alpha=0.01$ is considered as the frame synchronized case with $1 \%$ offset representing a nonideal synchronization. Similarly, $\alpha=0.99$ is assumed to be the frame synchronized case employing asymmetrical transmission, i.e., the base station in the middle cell is receiving, while the base stations in the adjacent cells in Fig. 5 are transmitting, and the mobiles in the middle cell are receiving, while the mobiles in the adjacent cells are transmitting. $\alpha=0.5$ gives the results for a nonsynchronized case with an offset time $t_{\text {off }}$ of half the duration of a time slot $t_{\text {slot }}$.

The simulation results are shown in Fig. 6. The uplink capacity degrades almost linearly as the portion of the base station-to-base station interference increases ( $\alpha$ increases) in Fig. 6(a). For only one user in each interfering cell, $68 \%$ of the initial capacity is left in the worst case $(\alpha=0.99)$. If there are 


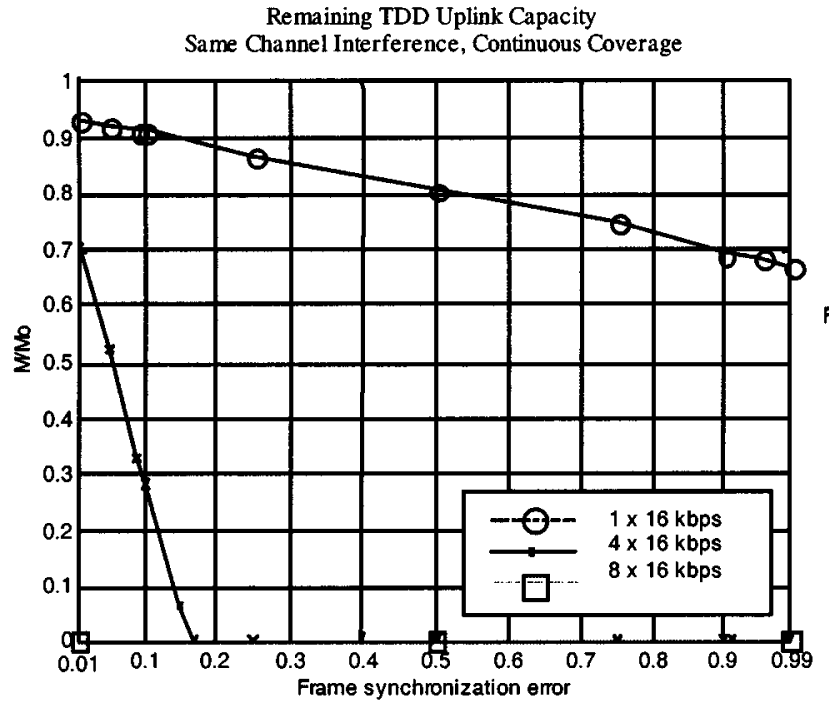

a) Uplink

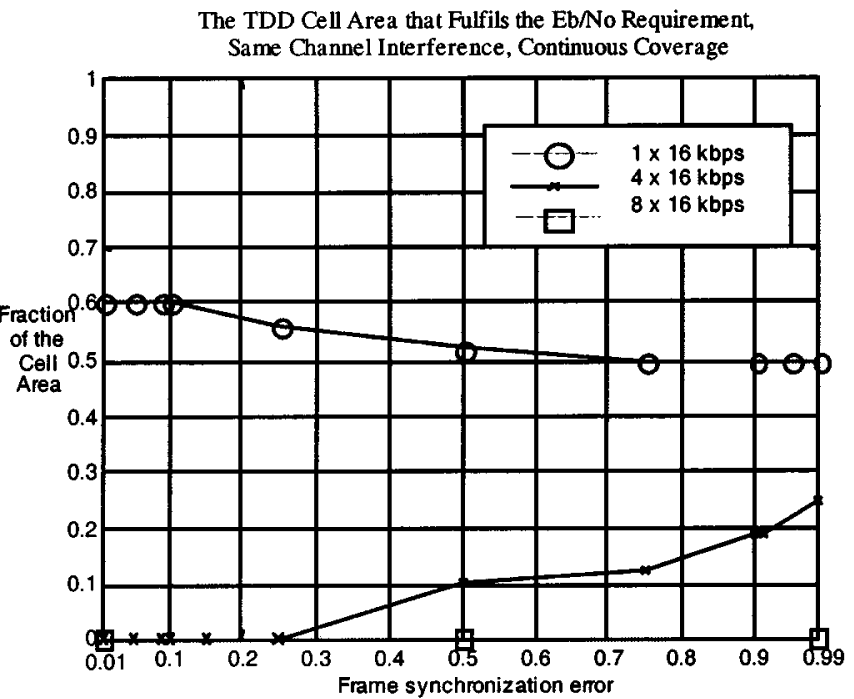

b) Downlink

Fig. 6. The effects of the same channel TDD-to-TDD interference with different loads in the interfering cells and different values of the frame synchronization error $\alpha$ ( $\alpha=0$ corresponds to frame synchronized cells with the same asymmetry in adjacent cells). (a) Uplink. (b) Downlink.

4 users per slot in the neighboring cells, $70 \%$ of the single cell capacity is left for the synchronized case $(\alpha=0.01)$. With $17 \%$ time offset or more, there is no capacity left in the middle cell. In this case, 8 users per slot cannot be supported in all cells even in the synchronized case. Note that joint detection receivers were not considered here in suppressing intra-cell interference.

For the downlink, the performance is not as sensitive to the frame synchronization. In the one-user case, the performance degrades only slightly, as the portion of the mobile-to-mobile interference increases ( $\alpha$ increases). The reason for this is that there is only one mobile which determines the base station transmission power in the interfering time slot. Therefore, the interference caused by the base station and the mobile station are approximately the same. Hence, the effects of a frame synchronization error become less crucial. However, the amount of interference from the base station and from the mobile station is different, because the distance between the edge of the middle cell and the interfering mobile can vary from almost zero to the outer diameter of the interfering cell whereas the base station is stationary. Hence, an interfering mobile can occasionally be fairly close to a mobile in the middle cell, and cause a high interference peak. Therefore, when there is only one user in the interfering cells, the impact of base station-to-mobile interference is less severe than that of mobile-to-mobile interference. However, the situation is reversed if more than one user is active. In this case, the transmission power of the base station for all connections is determined by the highest attenuation within the time slot. The overall transmission power of the base station becomes higher than that of the mobiles, and the impact of the mobiles located close to each other becomes less severe. A loading of $8 \times 16 \mathrm{~kb} / \mathrm{s}$ per time slot cannot be supported in the downlink in all cells at the same time.

The study shows that synchronization of the adjacent cells is clearly a key requirement for UTRA TDD, especially for the up- link performance. Time domain resource allocation by dynamic channel allocation can be used to reduce the inter-cell interference. As shown in Fig. 6, it is not possible to use a full loading in all times slots in adjacent cells, and dynamic channel allocation should be used to optimize the resource allocation. Dynamic channel allocation can reduce the interference between uplink and downlink as well.

\section{INTER-OPERATOR INTERFERENCE EVALUATION}

The scenario considered for evaluating the inter-operator interference is shown in Fig. 7. An adjacent channel protection of $30 \mathrm{~dB}$ is assumed. According to the latest 3GPP specifications [2], the adjacent channel protection is $33 \mathrm{~dB}$ for the terminal transmission. The difference of $3 \mathrm{~dB}$ does not cause practical differences to the results. For the base station transmission, the required adjacent channel protection values are higher, and the results in this paper are pessimistic with small base station separations. The results for adjacent channel interference for 4 users per slot and for 8 users per slot in the interfering TDD cells are shown in Figs. 8 and 9. The results for different synchronization scenarios are shown as a function of the base station separation. The bit rate is set to $16 \mathrm{~kb} / \mathrm{s}$.

In the uplink, collocation of the base stations (base station separation $\leq 5 \mathrm{~m}$ is considered as collocation) destroys the capacity in that time slot in the cell of interest. When the base stations are collocated, the UTRA TDD system works only if the operators' base stations are synchronized, i.e., $\alpha=0.01$. In the case of synchronized base stations, $42 \%$ of the single cell capacity is achieved, if the interfering cells on the adjacent frequency have 8 users per time slot. As the distance between the base stations becomes larger, the capacity figures improve. It can be seen in Figs. 8(a) and 9(a) that the capacity is relatively independent of the frame synchronization error of the two systems, if the distance between the base stations is $50-60 \mathrm{~m}$, 


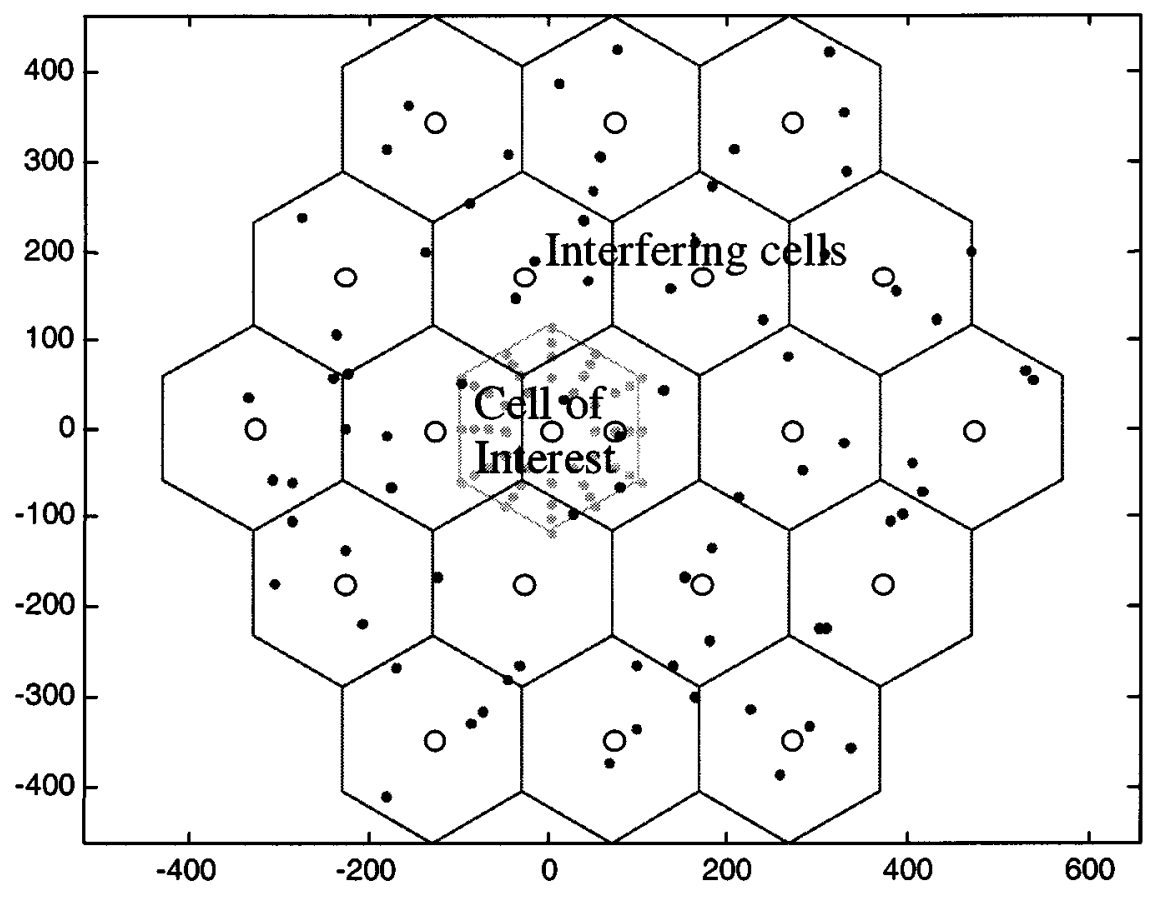

Base station

- Mobile station

Fig. 7. Simulation scenario for TDD-to-TDD adjacent channel interference.

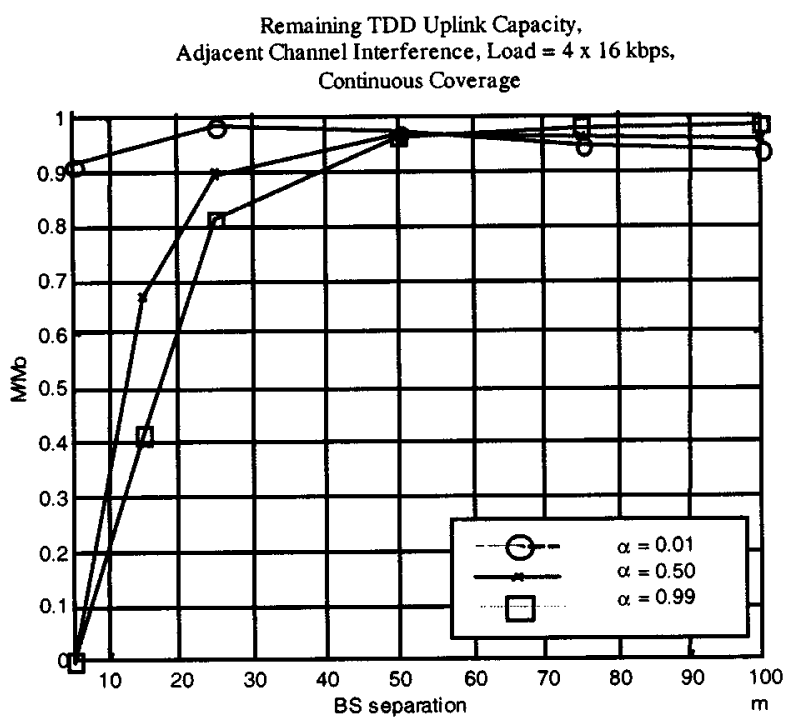

(a)

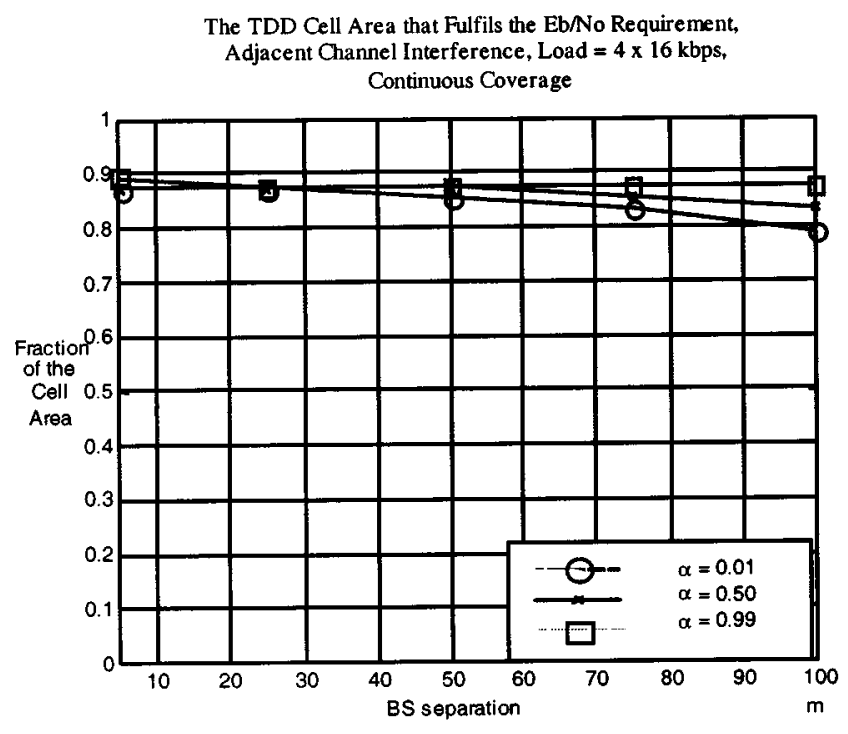

(b)

Fig. 8. The effects of the adjacent channel TDD-to-TDD interference with different base station separations and values of the frame synchronization error $\alpha$. The loading is $4 \times 16 \mathrm{~kb} / \mathrm{s}$. (a) Uplink. (b) Downlink.

i.e., approximately half of the cell radius $100 \mathrm{~m}$. Especially in Fig. 8(a), it can be easily seen that for distances smaller than this, more capacity is gained in the synchronized case $(\alpha=0.01)$; and for greater distances, it is beneficial to have the transmission in opposite directions, i.e., completely unsynchronized or asymmetrical with $\alpha=0.99$. The reason for this is that at the cell edge, the distance between the base stations is at its maximum, and hence the interference path is heavily attenuated, and the interference caused by the base station is at its minimum. In contrast to that, the transmission power levels of the mobiles located at the cell edge are high, and the distance between these mobiles and the base station is at its minimum. Therefore, the interference caused by the mobile stations is at its maximum.

In the downlink, the performance is not as strong a function of the base station separation as in uplink. At the cell edge, the performance is slightly poorer, but in this case the $E_{b} / N_{0}$ requirement is achieved for at least $70 \%$ of the cell area (full loading). The best downlink performance is achieved with $\alpha=0.99$. 


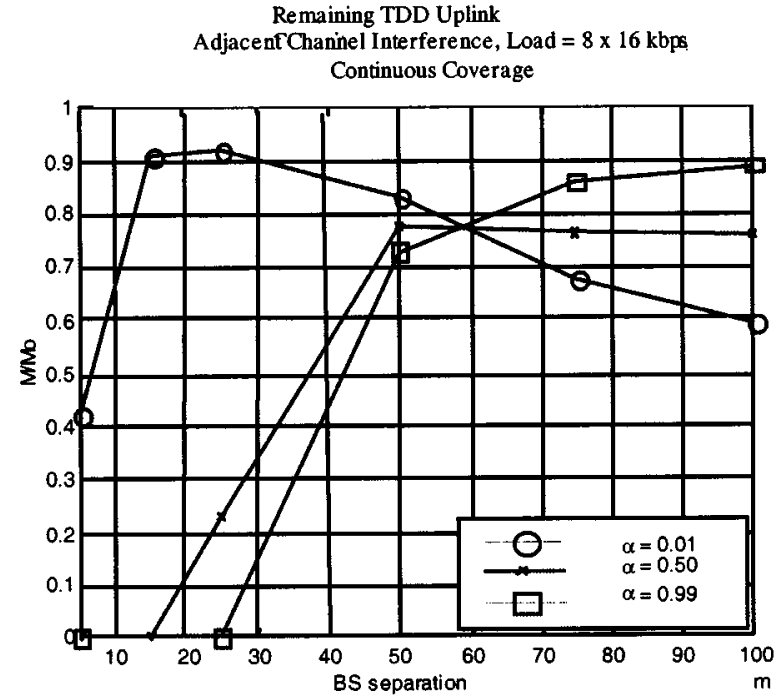

(a)

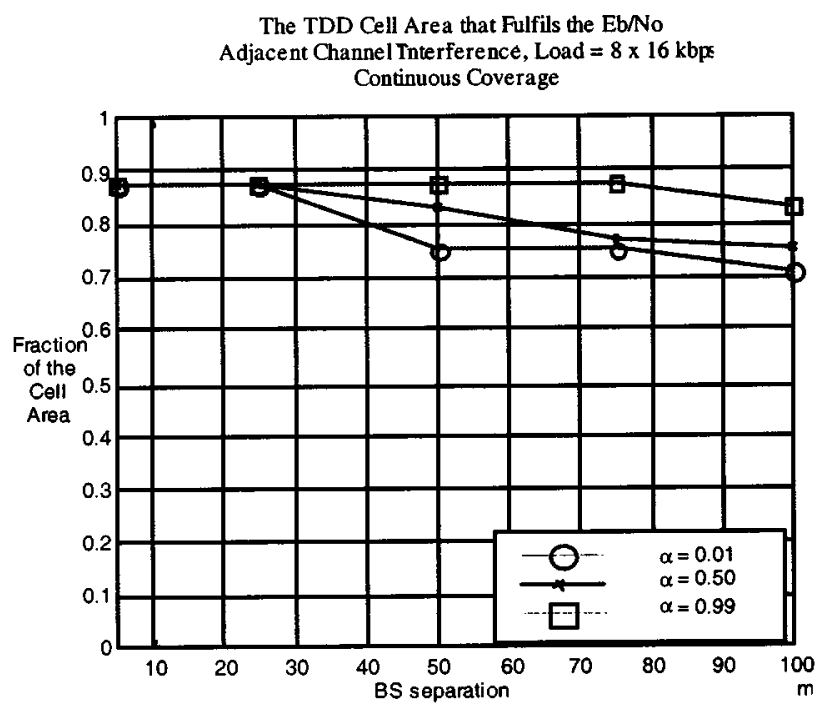

(b)

Fig. 9. The effects of the adjacent channel TDD-to-TDD interference with different base station separations and values of the frame synchronization error $\alpha$. The loading is $8 \times 16 \mathrm{~kb} / \mathrm{s}$. (a) Uplink. (b) Downlink.

The study shows that collocation of two operators UTRA TDD base stations is feasible only if the networks are synchronized, and if identical splitting between uplink and downlink is used. If the base stations are not collocated, the synchronization is not necessarily needed.

Dynamic channel allocation between two operators cannot be as optimal as within one operator's band because it is not controlled by one entity. The role of dynamic channel allocation between two operators is rather an interference escape mechanism.

The effect of the adjacent channel interference in UTRA TDD has been evaluated also in [6].

\section{SUMMARY}

The interference between uplink and downlink in UTRA TDD has been evaluated in this paper. This interference can occur between two terminals or between two base stations. The main problem is clearly the interference between two base stations. Therefore, it is beneficial to frame synchronize the adjacent TDD cells of the same operator. Careful network planning can be used to ease the interference problems between the base stations. The collocation of two operators UTRA TDD base stations in adjacent frequencies is feasible only if the networks are synchronized, and if identical splitting between uplink and downlink is used. If the base stations are not collocated, the synchronization is not necessarily needed. The solutions covered in this paper to tackle the interference problems in UTRA TDD include base station synchronization, network planning, and dynamic channel allocation.

\section{ACKNOWLEDGMENT}

The authors would like to thank Dr. G. Povey and H. Haas from the University of Edinburgh for their valuable contribu- tions and comments. The authors are also grateful for the useful comments given by the reviewers.

\section{REFERENCES}

[1] H. Holma and A. Toskala, Eds., WCDMA for UMTS_Radio Access for Third Generation Mobile Communications. New York: Wiley, Apr. 2000.

[2] 3GPP.. [Online]. Available: http://www.3gpp.org

[3] M. Haardt, A. Klein, R. Koehn, S. Oestreich, M. Purat, V. Sommer, and T. Ulrich, "The TD-CDMA based UTRA TDD mode," IEEE J. Select. Areas. Commun., vol. 18, pp. 1375-1385, Aug. 2000.

[4] H. Holma, G. Povery, and A. Toskala, "Evaluation of interference between uplink and downlink in UTRA/TDD," in Proc. VTC'99 Fall, Amsterdam, Netherlands, Sept. 19-22, 1999, pp. 2616-2620.

[5] S. Heikkinen, "System simulations for UMTS TDD and FDD modes," Master's thesis, Univ. Oulu, Finland/University of Edinburgh, Scotland, 1999. Available from Oulu Univ. Library, P.O. Box 4000, FIN-90014 Univ. Oulu, Finland.

[6] H. Haas and G. Povey, "The effect of adjacent channel interference on capacity in a hybrid TDMA/CDMA-TDD system using UTRA-TDD parameterss," in Proc. VTC'99 Fall, Amsterdam, Netherlands, Sept. 19-22, 1999, pp. 2616-2620.

[7] H. Haas, S. McLaughlin, and G. Povey, "The effects of interference between the TDD and FDD mode in UMTS at the boundary of $1920 \mathrm{MHz}$," ISSSTA 2000, submitted for publication.

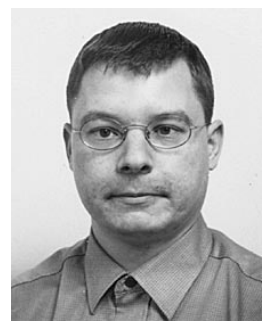

Harri Holma received the M.Sc. degree from Helsinki University of Technology in 1995.

He joined Nokia Research Center in 1994. Since 1994, he has been working with third-generation WCDMA air interface with special interest on radio network performance. In January 1998, he joined Nokia Networks and is currently working as a Senior Research Engineer with WCDMA radio network solutions. He has co-edited a book WCDMA for UMTS (Wiley, 2000) and authored three chapters in the book Wideband CDMA for Third Generation Mobile Communications (Norwood, MA: Artech House, 1998). 


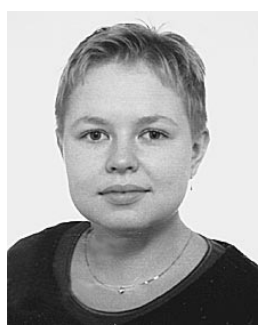

Sanna Heikkinen was born in Taivalkoski, Finland, in 1976. She received the M.Sc. degree in electrical engineering in 1999 from the University of Oulu, Finland.

She participated in TDD related research in co-operation with the University of Edinburgh, Scotland, and Nokia Corporation, Finland. Currently, she is employed by Kolster Oy Ab, Helsinki, Finland.

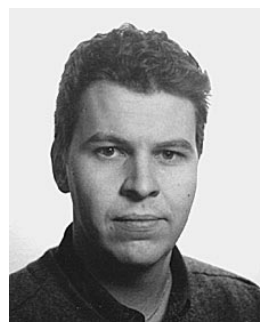

Otto-Aleksanteri Lehtinen received the M.Sc. degree from Helsinki University of Technology in 1999.

He joined Nokia Mobile Phones in 1997. Since 1998, he has been working with third-generation WCDMA air interface. Currently he is working as a Senior Design Engineer with WCDMA system solutions improving user equipment. He is a co-author of a chapter in the book WCDMA for UMTS (New York: Wiley, 2000).

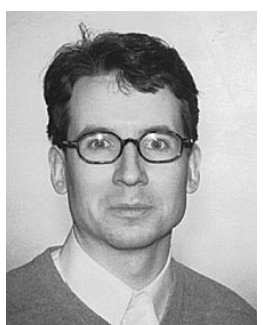

Antti Toskala received the M.Sc. degree.

He joined the Nokia Research Center in 1994, where he was involved with WCDMA system studies. In September 1995, he joined the ACTS FRAMES project. In later phase of the FRAMES project, he worked as the Team Leader for the work package which defined the FMA2 WCDMA concept. During 1997, he was working as a Senior Research Engineer and CDMA Specialist participating in the ETSI SMG2 UMTS standardization work. Currently, he is with Radio Access Systems at Nokia Networks working as Standardization Manager with WCDMA standardization and also working in 3GPP as Chairman for the TSG RAN WG1, the group being responsible for the physical layer of the WCDMA standard. He has large number of publications in the field and he is co-editor of the book WCDMA for UMTS (New York: Wiley, 2000). 